\title{
A REMARK ON METHOD IN TRANSFINITE ALGEBRA $\dagger$
}

\author{
BY MAX ZORN $\ddagger$
}

The theorems of Steinitz concerning algebraic closure and the degree of transcendence are barred, from the algebraic point of view, by the well-ordering theorem and its theory. We wish to show how, by introducing a certain axiom on sets of sets instead of the well-ordering theorem, one is enabled to make the proofs shorter and more algebraic. The proofs will be given in terms of the non-axiomatic standpoint of set theory.

Definition 1. A set $\mathfrak{B}=\{B\}$ of sets $B$ is called a chain, if for every two sets $B_{1}, B_{2}$, either $B_{1} \supset B_{2}$ or $B_{2} \supset B_{1}$.

Definition 2. A set $\mathfrak{A}$ of sets $A$ is said to be closed (rightclosed), if it contains the union $\sum_{\mathbb{R} \ni B} B$ of every chain $\mathfrak{B}$ contained in $\mathfrak{A}$. form.

Then our maximum principle is expressible in the following

(MP). In a closed set $\mathfrak{A}$ of sets $A$ there exists at least one, $A^{*}$, not contained as a proper subset in any other $A \in \mathfrak{A}$.

Applications. I. Let $\Re$ be a ring with a unity element 1 ; let $\mathfrak{A}$ be the set of all ideals $\mathfrak{a}$ (i) not containing 1 as an element, (ii) containing a certain ideal $\mathfrak{r} \neq \mathfrak{R}$. The set $\mathfrak{A}$ is obviously closed; the maximum principle implies the existence of a maximal ideal $\mathfrak{p}$ with $\mathfrak{r} \subseteq \mathfrak{p} \subset \Re \neq \mathfrak{p}$; this ideal is a prime ideal and the residue class ring $\Re / p$ is a field.

II. If $k$ is a real field, that is, a field such that no sum of squares vanishes unless all the squares vanish, and $K$ is an arbitrary algebraical extension, then the set of all real fields between $k$ and $K$ is closed, so the MP assures the existence of a maximal real field between $k$ and $K$. In particular, if $K$ is algebraically closed, we obtain a real closure of $k$.

III. Let $K$ be an arbitrary field extension of $k$. A set of $K$ elements $\{a\}$ is said to be algebraically (respectively, linearly) independent, if no finite subset $a_{1}, a_{2}, a_{3}, \cdots, a_{n}$ satisfies an

$\dagger$ Presented to the Society, October 27, 1934.

$\ddagger$ Sterling Fellow. 
algebraic (linear) relation with coefficients in $k$ (not all vanishing). The set of all these independent sets is closed; a maximal independent system, which exists as a consequence of the MP, is a basis for $K / k$ with respect to algebraic (linear) dependence. (The theorems concerning dependence are consequences of the dependence axioms $†$ only.)

We shall first prove the existence of an algebraic, algebraically closed field extension. Indeed, we shall construct for each set of polynomials $\{p(x)\}$ with coefficients in $k$ a minimal decomposition field. (Let $p$ have the highest coefficient 1 and degree $n_{p}$.)

We form the field $k^{\prime}$ of all rational functions in $x$ over $k$. Over $k^{\prime}$ we consider the domain of all polynomials in the following set of indeterminates: $y_{p, i}$, the first index $p$ ranging over all polynomials of our set, the second over the integers from 1 to $n_{p}$.

In this domain of integrity let us take the ideal generated by the polynomials

$$
\left\{p(x)-\left(x-y_{p, 1}\right)\left(x-y_{p, 2}\right) \cdots\left(x-y_{p, n_{p}}\right)\right\} .
$$

Suppose that this ideal does not contain the number 1 . Then we have a prime ideal containing it, and the residue classes of the indeterminates $y$ generate a minimal field over $k$, in which all polynomials $p(x)$ are completely decomposed.

If now this ideal should contain the unity element, we should have a representation of 1 as a linear combination of a finite set of differences $p(x)-\left(x-y_{p, 1}\right) \cdots$ with coefficients rational in $x$ and integral in the $y$ 's. This relation in indeterminates $y$ gives the contradiction 1 equal to 0 , if we substitute for the indeterminates the roots of the finite set of polynomials $p$ occurring in the relation; these roots exist in accordance with elementary theorems in a suitable field extension.

Let $K_{1}, K_{2}$ be two algebraic extensions of $k$, which are minimal decomposition fields for the set of polynomials $\{p(x)\}$ over $k$. We consider the partial realizations of the uniqueness theorem, that is, the isomorphisms between fields $K_{1}{ }^{2}$ and $K_{2}{ }^{2}$, which are the identity in the common subfield $k, K_{i}{ }^{2}$ lying in $K_{i}$. The function tables of these isomorphisms, considered as sets of pairs, form a closed system (of sets of pairs); therefore a maximal one must exist in accordance with MP.

† Van der Waerden, Moderne Algebra, vol. 1, p. 204. 
This maximal isomorphism must range over the entire fields $K_{i}$. For let $K_{i}{ }^{0}$ be the fields connected by the maximal isomorphism. Take an arbitrary polynomial $p(x)$ in the set $\{p\}$. The minimal decomposition fields for this polynomial $p(x)$ over $K_{i}{ }^{0}$, generated by adjoining respectively the roots in $K_{i}$, are isomorphic under an isomorphism which continues our maximal isomorphism between $K_{1}^{0}$ and $K_{2}{ }^{0}$. (As in the case of the existence these proofs are based on the corresponding finite theorems; precisely as in the proof dealing with the degree of transcendence.) Hence the decomposition fields reduce to $K_{i}{ }^{0} ; K_{i}{ }^{0}$ decomposes all polynomials of the set and is therefore identical with $K_{i}$; our maximal isomorphism yields the uniqueness of the minimal decomposition field, in particular, the uniqueness of algebraic closure.

Finally we prove the existence of the degree of transcendence. Let $\{x\}$ and $\{y\}$ be two bases of $K / k$. Once more we consider the partial realizations of our theorem. Namely, we take a $(1,1)$ correspondence between subsets $\{x\}^{\prime}$ and $\{y\}^{\prime}$ of $\{x\}$ and $\{y\}$, where $\{x\}^{\prime}$ and $\{y\}^{\prime}$ are dependent.

The function tables of these correspondences, considered as sets of pairs, form a closed system. We shall prove by the corresponding finite theorem concerning degree of transcendence and the axiom of choice that a maximal correspondence of this kind which exists in accordance to MP must range over the entire bases $\{x\}$ and $\{y\}$. Take a correspondence with $\{x\}^{\prime}$ different from $\{x\}$. Take a finite set of $x$ 's not contained in $\{x\}^{\prime}$. This will depend on $\{y\}^{\prime}$ and a certain finite set of $y$ 's which is not contained in $\{y\}^{\prime}$. This finite set of $y^{\prime}$ s depends on $\{x\}^{\prime}$, the arbitrarily chosen finite set of $x$ 's and a certain other finite set of new $x$ 's; proceeding in zigzag we obtain (choice axiom) two sequences of new $x$ 's and $y$ 's; we may proceed similarly, in case of a finite number (finite theorem) or an infinite (denumerable) set. It is not necessary to show the utility of the MP in further algebraic or analytic examples.

In another paper I shall discuss the relations between MP, the axiom of choice, and the well-ordering theorem. I shall show that they are equivalent if the axiom yielding the set of all subsets of a set is available. It seems that without this axiom the MP is weaker than the well-ordering theorem; the situation may be studied by means of other axioms.

In the present paper all the axioms concerning sets are used; 
in the case of algebraic closure there is a stronger result of Hollkott (Hamburg): the axiom of choice is sufficient for the existence and uniqueness of algebraic closure.

An essential simplification is made possible for Baer's $\dagger$ theory of the degree of algebraic extensions. I plan to show elsewhere how the generalized continuum hypothesis may be avoided.

Yale University

\section{CONCERNING TWO INTERNAL PROPERTIES OF PLANE CONTINUA}

BY R. E. BASYE

Theorem 1 below was suggested to me by R. L. Moore. Theorem 2 is an extension of Kuratowski's result $\$$ that if three compact plane continua have a point in common and their sum separates a point $A$ from a point $B$ in the plane, then there exists a pair of these continua whose sum separates $A$ from $B$ in the plane. Another extension of this result along combinatorial lines has been given\| by Cech.

TheORem 1. Let $H$ and $K$ be two mutually exclusive and closed subsets of a compact continuum $M$ which lies in the plane. If for each pair of points $A$ and $B$ in $H$ and $K$, respectively, there exists a finite collection $\Gamma_{A B}$ of continua in $M$ such that $\Gamma_{A B}^{*}$ separates $A$ from $B$ in $M$, then there exists a finite collection $\Gamma$ of continua in $M$ such that $\Gamma^{*}$ separates $H$ from $K$ in $M$.

Let $\epsilon_{1}, \epsilon_{2}, \cdots$, be a sequence of positive numbers converging monotonically to zero, with $\epsilon_{1}$ less than half the distance from $H$ to $K$. For each $i$ let $D_{H}^{i}$ be a domain containing $H$ such that (1) the boundary $\beta_{H}^{i}$ of $D_{H}^{i}$ is the sum of a finite number of mutually exclusive simple closed curves, and (2) each point of

$\dagger$ Eine Anwendung der Kontinuumhypothese in der Algebra. Journal für Mathematik, vol. 162.

$\ddagger$ Presented to the Society, April 6, 1935, under a somewhat different title.

$\S$ Kuratowski, Théorème sur trois continus, Monatshefte für Mathematik und Physik, vol. 36 (1929), pp. 77-80.

$\|$ E. Čech, Trois thêorèmes sur l'homologie, Publications de la Faculté des Sciences de L'Université Masaryk, No. 144, 1931, pp. 1-21. 